\title{
Article
}

\section{Can child safeguarding training be improved?: findings of a multidisciplinary audit}

Patrick, A., Kandiah, T. and Welbury, Richard

Available at http://clok.uclan.ac.uk/31748/

Patrick, A., Kandiah, T. and Welbury, Richard ORCID: 0000-0002-9322-2440

(2020) Can child safeguarding training be improved?: findings of a multidisciplinary audit. European Archives of Paediatric Dentistry . ISSN 18186300

It is advisable to refer to the publisher's version if you intend to cite from the work. http://dx.doi.org/10.1007/s40368-020-00513-1

For more information about UCLan's research in this area go to http://www.uclan.ac.uk/researchgroups/ and search for < name of research Group>.

For information about Research generally at UCLan please go to http://www.uclan.ac.uk/research/

All outputs in CLoK are protected by Intellectual Property Rights law, including Copyright law. Copyright, IPR and Moral Rights for the works on this site are retained by the individual authors and/or other copyright owners. Terms and conditions for use of this material are defined in the policies page.

\section{CLoK}

Central Lancashire online Knowledge www.clok.uclan.ac.uk

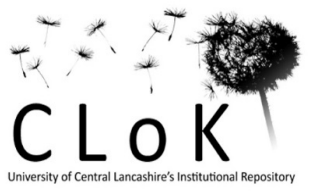


Can child safeguarding training be improved? Findings of a multidisciplinary audit.

Patrick A

Specialty Training Registrar Oral Surgery

Eastman Dental Hospital, University College London Hospitals NHS Trust, UK

East Surrey Hospital, Surrey and Sussex Healthcare NHS Trust, UK

Correspondence - amy.patrick@nhs.net

Kandiah T

Clinical Lead \& Consultant Paediatric Dentistry Dental and Maxillofacial Dept

East Surrey Hospital, Surrey and Sussex Healthcare (SASH) NHS Trust, UK

Welbury R

Professor / Honorary Consultant in Paediatric Dentistry

University of Central Lancashire, Preston, UK. 
Abstract

Introduction

Child protection has become a very poignant subject in the UK. The Laming report into the death of Victoria Climbié led to the implementation of multidisciplinary safeguarding policies and training for healthcare professionals, social services staff and police. However many dental practitioners, as well as other healthcare professionals, have little confidence when reporting their concerns and reducing the 'gap' between suspicion and reporting (Laming, 2003).

Our audit aimed to identify and address the barriers of reporting safeguarding concerns amongst the hospital team.

\section{Materials and Methods}

Questionnaires were distributed to staff at SASH NHS Trust with a valid Safeguarding Children Level 3 certificate in order to assess their knowledge of safeguarding children. Changes to traditional safeguarding training (Group 1) were implemented to include a broader range of speakers, each speaking for shorter times with more personal and focused presentations (Groups 2 and 3). Three cohorts of participants were assessed including healthcare assistants, nurses, dentists, and doctors.

Results

Group 1 ( $n=100$ ) reported $64 \%$ experience of reporting compared with group $2(n=100) 43 \%$ and group $3(n=76) 51 \%$. Confidence was higher in those with more experience of child protection. The most common barrier was uncertainty of diagnosis. More barriers to reporting existed in the new style of training in groups 2 and 3 . The scenarios were answered with appropriate concern and reporting by groups 1 and 2, however less for group 3 .

\section{Discussion}

Experienced and trained practitioners are more likely to refer children to child protection teams when they have concerns of abuse or neglect. Personalising training was shown to be less effective and the focus should be more on diagnosis and local protocols. Early training from undergraduate level was a unanimous request.

\section{Conclusion}

Focused training on diagnosis and local protocols, accompanied by bespoke teaching for specific specialties would be the most constructive tool for safeguarding children. Exploring modern methods such as simulation based training could be effective. Structured forms and local policies that are familiar to clinicians prevent omissions and encourage professional awareness.

Key words: safeguarding child protection abuse neglect training 


\section{Main document}

Introduction

Child protection has become a very poignant subject in the UK over the last few years, with policies implemented by the Department of Health (DOH), British Dental Association (BDA), Royal College of Paediatrics and Child Health (RCPCH), and the General Medical Council (GMC). The National Society for the Prevention of Cruelty to Children (NSPCC) estimates that in England over 50,000 children were in need of a protection plan in 2018 (a number that has been increasing each year) and that between 1 and 2 children die every week as a result of neglect or abuse despite these measures being put into place (NSPCC, 2019). Ten years ago, the Laming progress report (2009) on his original 2003 report published many recommendations to encourage a change in culture and raise the profile of safeguarding children; this included revising the guidance 'Working together to safeguard children' by making it clearer and more comprehensive, and importantly focussing on preventive services and early intervention. The advice to the DOH and the Department for Children was to ensure adequate training was provided for all staff so that no child was able to leave an emergency department whilst there were concerns for their safety (Laming, 2009). This policy was updated in 2018 with significant revisions including assessing need and providing help, organisational responsibilities, multi-agency safeguarding arrangements, local and national child safeguarding practice reviews, and child death reviews (Department for Education, 2018).

The legislation around training in the UK depends on the level of contact a professional has with children and certain levels of training must be achieved for different people. General dental practitioners (GDPs) routinely (but not exclusively) treat children and therefore the guidance would require them to attain Level 2 training, however some dental practitioners with significant responsibility may require level 3 training and this will be determined locally within larger organisations (including hospital, community-based specialist services, paediatric dentistry or other relevant dental specialties such as orthodontics) based on an assessment of need and risk. This is outlined in the 'toolkit for dental teams' (PHE, 2019) which requires an individual to have a minimum of 3 hours of training, reviewed annually and refreshed every 3 years. Many studies have looked at the training received and what influence this has had on their confidence, knowledge, and attitude to safeguarding.

Evidence of training either at dental undergraduate or postgraduate level is variable in many countries, with different teaching modalities utilised including one to one training, seminars, lectures or multiagency involvement (Harris 2009, Laud 2013, Cairns 2005). Figures show that up to 97\% of dentists strongly request further training (Harris 2009, Laud 2013, Al-Dabaan 2014, Bannon 2003, Welbury 2003, Al-Habsi 2009). The British Society for Paediatric Dentistry (BSPD) recommends mandatory training programmes for undergraduates, foundation dentists, specialty dentists, and specialist trainees (Harris, 2009).

Published studies have investigated the effectiveness of safeguarding training and although the numbers in each study differ significantly, all come to the same conclusion; many dentists have safeguarding concerns about children but few actually make a referral to the child protection team (Harris 2009, AL-Habsi 2009, Cairns 2005, Lazenbatt 2006). Some $38 \%$ of dentists do not refer when 
they have safeguarding concerns so it is vital that reasons for this are identified. The most common barrier found was uncertainty of the diagnosis, followed by fear of violence to the child, fear of litigation, and concerns about violence toward the dental team (Harris 2009, Laud 2013, Welbury 2003).

The dental evidence clearly shows a lack of confidence when it comes to safeguarding and child protection protocols. Barriers have been identified and yet year on year we haven't closed the gap significantly between concern and referral.

Aims

An audit was undertaken at Surrey and Sussex Healthcare NHS Trust (SASH) to identify and address barriers to reporting.

\section{Materials and Methods}

In 2017 a questionnaire (based on a previously study (Harris, 2009)) was sent to all current members of staff at SASH that have already undertaken level 3 child protection training (Group 1). Staff members included healthcare practitioners, nurses, and doctors of all experiences. The questionnaire focused on experience, confidence and attitude, barriers to reporting, with scenarios to evaluate their actions. Based on the results, barriers were identified and appropriate changes made to the programme for subsequent attendees (Groups 2 and 3). More speakers with shorter presentation times to retain audience focus, allow sharing of a broader range of information and allow speakers to share their personal safeguarding experiences. Other changes included provision of bespoke training specific for individual clinical teams ensuring an understanding relevant to specific clinical scenarios and involving the protocols and 'go-to' people for that clinical team. A second (Group 2) and third (Group 3) cohort were then given the same questionnaire as Group 1 but after receiving their updated safeguarding training day format. All three groups had a similar demographic.

Results

Group 1 had 104 responses with 4 exclusions (2 gave a written report rather than completing the questionnaire, 1 didn't recall their training and 1 returned an unanswered questionnaire). Group 2 had 100 responses and Group 3 some 76 responses.

Experience of reporting to a child protection team was $64 \%$ ( $n=64$ Group1), $43 \%$ ( $n=43$ Group2) and $51 \%$ ( $n=38$ Group3). See Figure 1. 


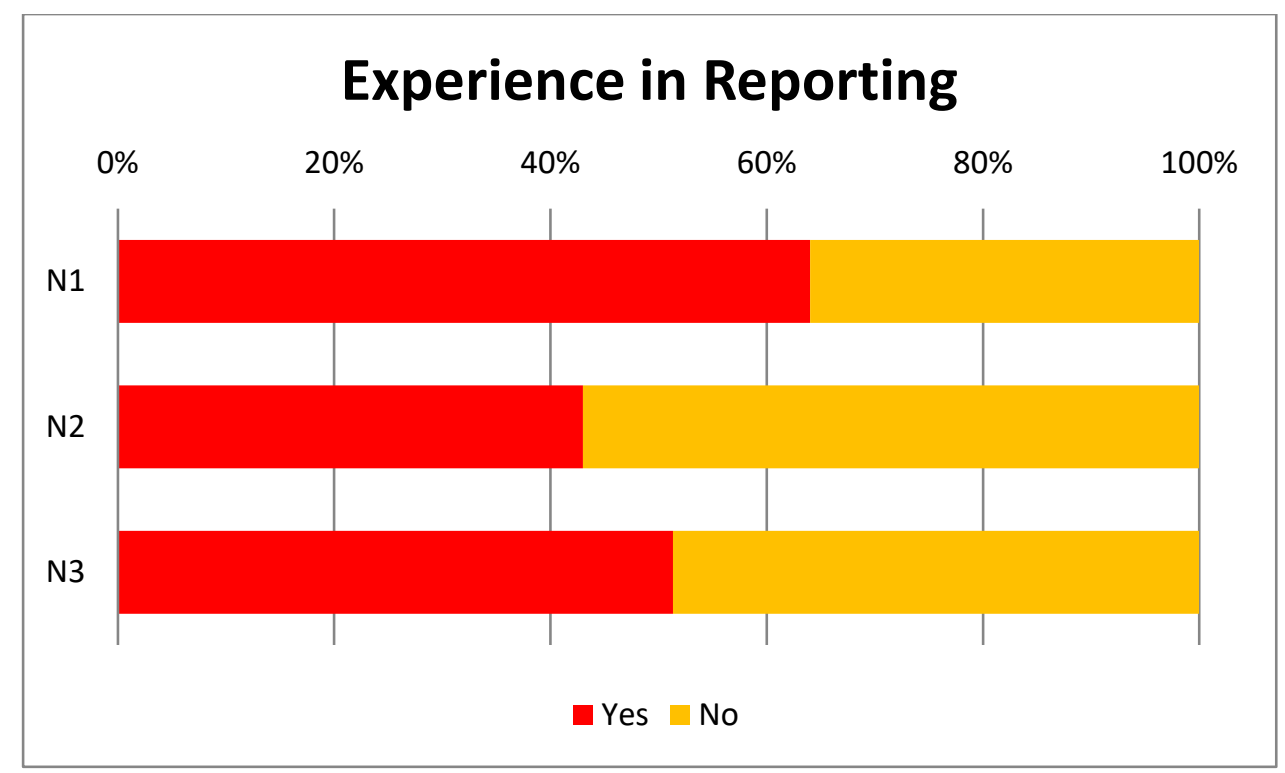

Figure 1 - Experience in reporting concerns to child protection teams.

Group 1 generally felt they had adequate safeguarding training and were confident that child abuse was preventable using the guidance. The majority of respondents from groups 2 and 3 reported training was adequate although a small number thought they required additional training to feel more confident to engage with safeguarding guidelines. Two of the respondents in group 1 felt training was not required as often as legislation dictates and that content was often too graphic and upsetting. In all groups more than $95 \%$ said they did not receive any information about follow up of children after information sharing following a referral and for this reason could not be certain if the child or families had benefitted from the intervention.

Confidence in reporting to child protection teams was moderate to high for all groups (measured on an ordinal scale), but group 1 were most confident. Ensuring children from homes with known domestic violence are assessed (a known risk factor for abuse and neglect) reached agreement in groups 1 and 2, but the question was removed for group 3 as free text feedback from groups 1 and 2 revealed poor understanding of the issue. Physical injury management was variable within the groups but was likely to be highest amongst emergency department, paediatric, and dental staff.

Overall group 2 showed a left skew with all safeguarding areas showing less confidence in comparison to other groups. Group 3 had a mid-range result between groups 1 and 3 (see figures 24). 

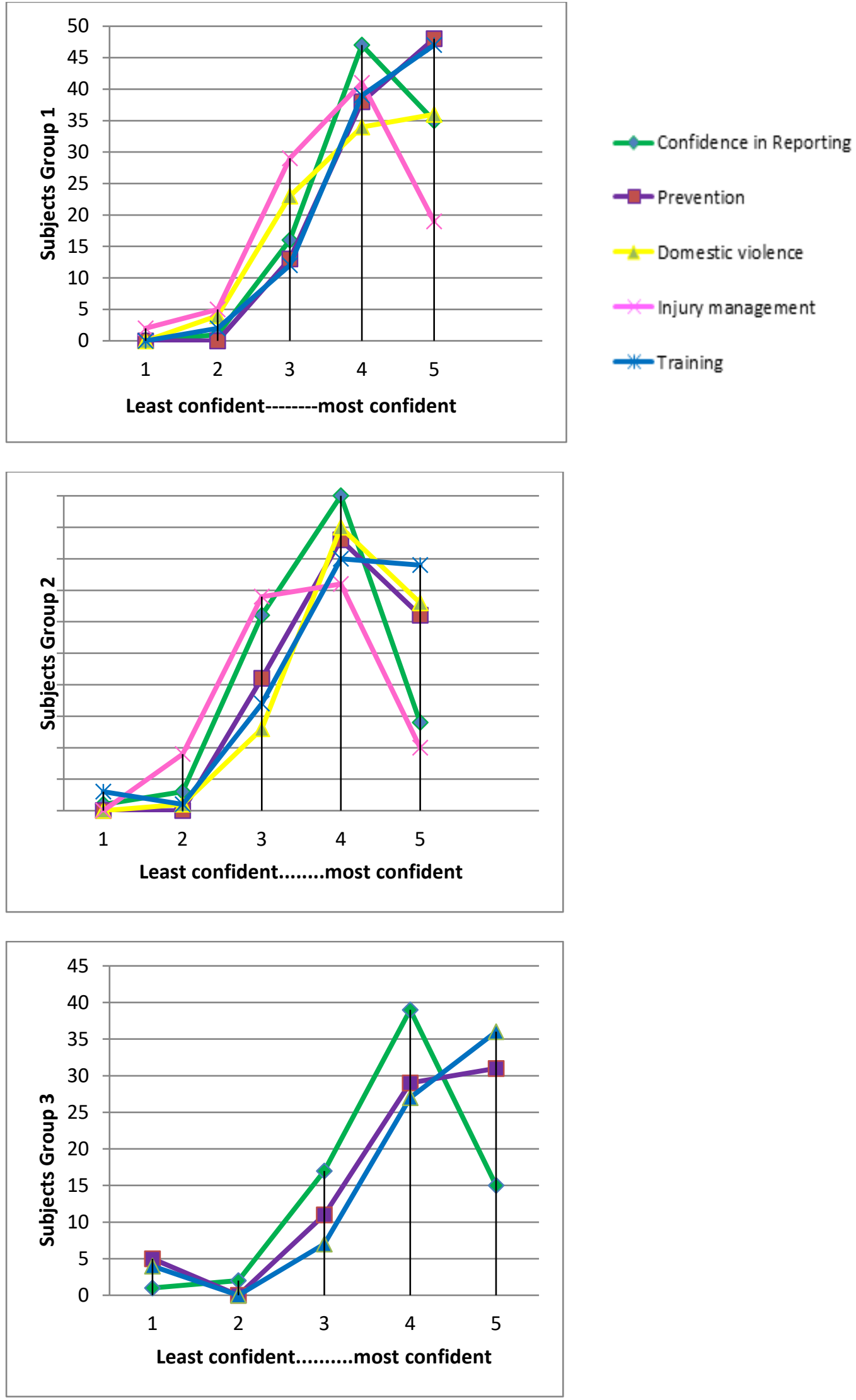

Figure 2 (group 1), Figure 3 (group 2), Figure 4 (group 3) - Confidence and attitude regarding safeguarding children 
The most common barriers to reporting included being unsure of the diagnosis and concerns for the safety of the child and the professionals themselves (see figure 5).

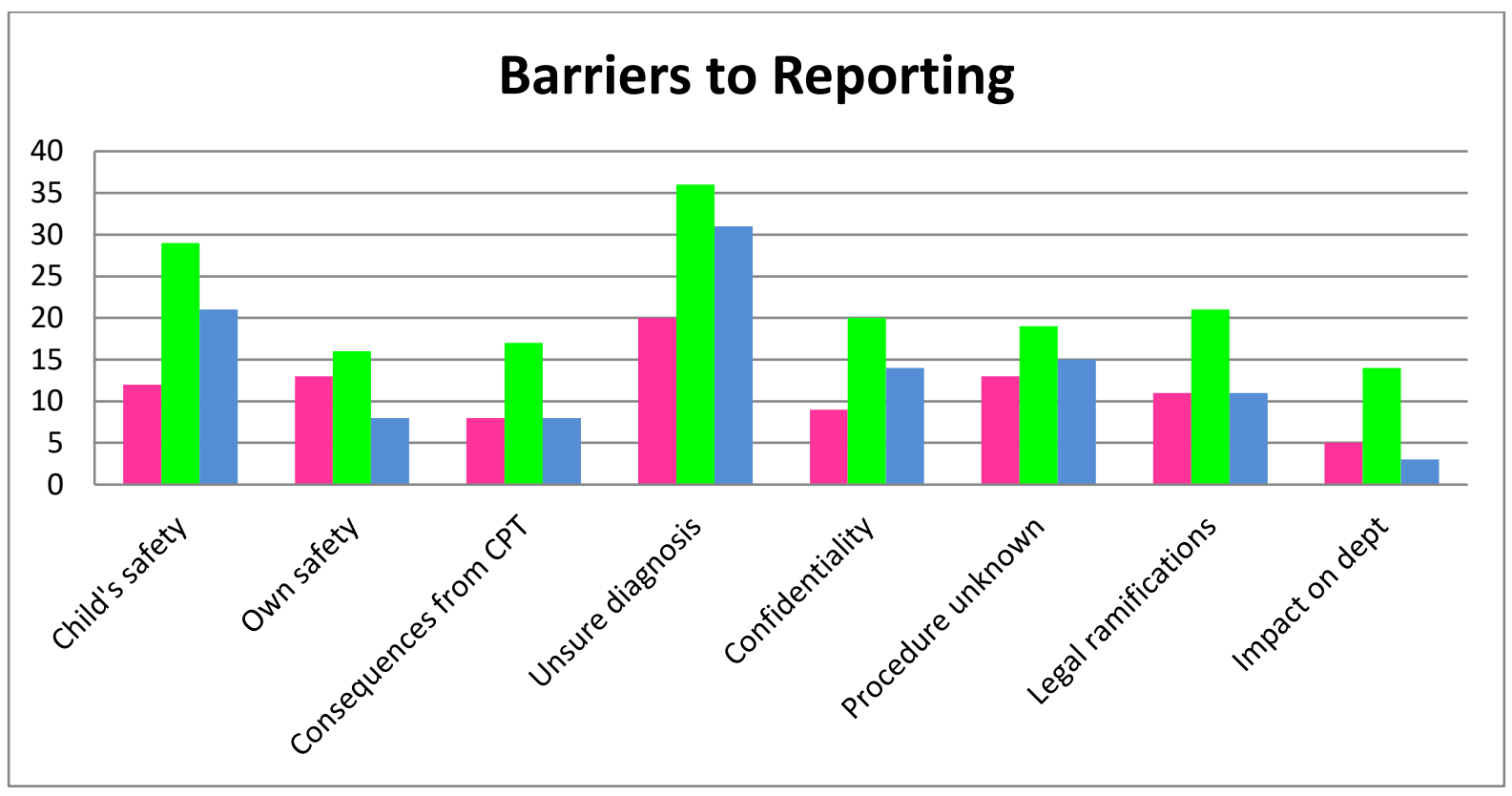

Figure 5 -Barriers to reporting for groups 1, 2, 3.

Two scenarios were given at the end of the questionnaire asking participants if they had a concern, and if they would report it. Both scenarios were based on real child cases that were put on a child protection plan. Of the first scenario groups 1 and 2 showed 100\% ( $n=100)$ concern and $98 \%(n=98)$ reporting. Group 3 showed $99 \%(n=75)$ concern and $94 \%(n=71)$ reporting. The second scenario (which was less ambiguous) showed 100\% ( $n=100$ ) concern and reporting for groups 1 and 2, and 99\% ( $n=75)$ concern and $96 \%$ ( $n=73)$ reporting for group 3.

\section{Discussion}

Group 1 was a larger sample who had received training within the last 3 years and included a variety of staff ranging from healthcare assistants to senior consultants. Groups 2 and 3 completed their questionnaires after the 'new style' training day and for many it could their first exposure to training, and many may only have relatively recently joined the trust or indeed their profession (within 6-36 months). In comparison group 1 may have included longer term staff within the trust; respondents in this group would have been at the trust between 6 months and 3 years to have a valid certificate, and this may account for the higher level of experience that was reported. Groups 2 and 3 may include new staff on their mandatory training for example junior doctors that rotate every 4-6 months; there are many instances where they may not be dealing with children or emergency admissions during this time. The range of jobs and grades were similar in all three groups, however 
even for those that are new to the trust it can be assumed that a new paediatric consultant or senior nurse has had prior training and this encounter would not be their first.

Welbury et al (2003) investigated the perceived role of the GDP regarding child protection through interviews with GDPs, local dental committees, social services, and paediatric and community dental teams, and found that only those with more than 15 years of experience had significantly higher confidence levels regarding all aspects of safeguarding children including referrals. This could account for the reduced confidence in reporting in groups 2 and 3.

Another possibility is that within 3 years after training, complacency has set in, and the newly trained responders are now aware of the difficulties in diagnosis, the barriers that may arise and the complexities of reporting. Even though the procedure for reporting has been explained it may be that the construct as a whole is rather intimidating and individuals may realise how much they do not know. This would explain why the number of barriers that exist for the second cohort has increased. The most common barrier for all groups was being unsure of the diagnosis for example GDPs felt less confident when diagnosing emotional and sexual abuse compared with physical (Welbury 2003). This is mirrored in the results regarding competence to treat any injuries. Dentists are in a good position to see physical abuse as they are performing an examination and evidence shows that $59 \%$ of abused children show signs on the head, face, mouth, or neck (Cairns 2005). Interestingly, although this has been identified as the most common barrier it is not for the GDP to make the diagnosis of child abuse, it is the role of the safeguarding team alongside paediatricians and social services. The main role of the dental team is to raise concern and to know where and to whom to share this concern with.

The BSPD advocate training from undergraduate level, and regular training throughout specialist training (Harris 2009). Out with medicine feedback from students on Initial Teacher Training (ITT) felt their knowledge of child protection procedures was poor. As a result the NSPCC designed education programmes with very positive results (Baginsky 2005).

The Social Care Institute for Excellence (SCIE) trialled several methods of training and recommended that a multi-agency systems approach would be the most beneficial (Fish 2008). This would accompany serious case reviews and common assessment frameworks to ensure all aspects for each child were addressed. This is also a common theme when looking at the evidence regarding GDPs attitudes to reporting. Many requested an increase in communication with medical specialties, social professionals, and local safeguarding children boards. However Bannon et al (2003) described ineffective communication within child protection pathways and concluded that abuse should be treated like any other disease; concern should be investigated, and any findings managed via the appropriate pathways and this could only be achieved with additional training. There is other evidence however that despite investigating several methods, no benefit was found from interagency training (Charles 2009). There is good evidence however that simulation based training in smaller groups is readily transferable to clinical practice (Jackson 2017, Wyllie 2019). Alternative improvements have been suggested from a systematic review of child protection training, for example putting check lists and structured forms into place. Improving documentation not only ensures all information has been gathered and shared appropriately but it also enhances professional awareness (Carter 2006).This correlates with the BSPD policy that advises local policies 
for neglect to include documented follow up plans and protocols for 'was not brought' (WNB) cases (Harris 2009).

Overall, the results in our 3 groups have one clear correlation. The more experience one has of child protection cases, the more confident one is to report concern and the more likelihood one is to have fewer barriers to reporting. The changes made to the training programme were designed to make it more personal, allowing for exploration of different avenues, and making headline points whilst keeping focus; rather than listening to long lectures. Despite these changes the results for all areas showed a decline. This was particularly concerning regarding the scenarios; group 3 did not all note obvious concern and even fewer reported concern. This indicated that the new methods for teaching were less effective than the original programme.

Guidelines dictate that child protection training must occur every three years. The evidence advising a multidisciplinary and personable approach appears to have result in substandard training in this audit with participants feeling less confident. Rather than diluting the core messages during the formal training day, we recommend that the focus should primarily be one of didactic teaching using local policies and problem based learning together with the national and local case reports. Annual refresher training is an excellent opportunity to incorporate external speakers without compromising the educational aspects of the didactic teaching.

\section{Conclusion}

Legislation and guidance regarding safeguarding children training is becoming more targeted with the Department of Health and other organisations publishing guidance detailing the pathways that should be followed. Despite this, healthcare practitioners still do not feel confident when they have concerns; partly due to hesitancy regarding diagnosis but also a lack of knowledge of local policies for reporting.

Training is essential to impart this knowledge to any person working with children, and these training days should be focused on diagnosis and protocols for reporting. Additional bespoke training to specialty departments can then be much shorter and focus on their particular specialty, for example dental practitioner and oral and maxillofacial departments can have cases focused on oral and head and neck injuries, and in addition their local policies can be discussed. Showing the pathway that individual cases have taken will broaden the training experience of participants

Other forms of training, for example simulation-based should be explored to prevent regular training from becoming tiresome whilst instilling a robust current practice.

Structured forms prevent participants from missing important points during information gathering, enhance professional awareness, and ensure appropriate information sharing. This practice can be used from initial consultation, routine follow ups and through to 'Was Not Brought' (WNB) policies and follow up of safeguarded children, whether from new referrals or previously initiated child protection plans.

Ultimately, in spite of child protection training being harrowing to some, it is the responsibility of every professional that has contact with a child to ensure they take a full history, document any concerns, share information when appropriate, whilst hopefully feeling comfortable with each step 
of these processes. Only when this is achieved will we be able to help more vulnerable children and close the gap between concern and reporting.

References

Al-Dabaan R, Newton JT, Asimakopoulou K. Knowledge, attitudes, and experience of dentists living in Saudi Arabia toward child abuse and neglect (2014) The Saudi Dental Journal Vol 26 Pg 79-87

Al-Habsi SA, Roberts GJ, Attari N and Parekh S. A survey of attitudes, knowledge and practice of dentists in London towards child protection. Are children receiving dental treatment at the Eastman Dental Hospital likely to be on the child protection register? British Dental Journal (2009) Vol 206: E7

Baginsky $\mathrm{M}$ and Macpherson P. Training teachers to safeguard children: developing a consistent approach (2005) Child Abuse Review Vol 14 Issue 5 Pg 317-330

Bannon MJ, Carter YH. Paediatricians and child protection: the need for effective education and training (2003) Archives of Disease in Childhood Vol 88 Pg 560-562

British Dental Association. Child Protection and the Dental Team (2006 revised 2009). Available at: https://bda.org/childprotection/Resources/Documents/Childprotectionandthedentalteam v1 4 No v09.pdf Accessed June 2019.

Cairns AM, Mok JY, Welbury RR. The dental practitioner and child protection in Scotland (2005) British Dental Journal Vol 199 Pg 517-520

Cairns AM, Mok JY, Welbury RR. Injuries to the head, face, mouth, and neck in physically abused children in a community setting (2005) International Journal of Paediatric Dentistry Vol 15 Issue $5 \mathrm{Pg}$ $310-318$

Carter YH, Bannon MJ, Limbert C, et al. Improving child protection: a systematic review of training and procedural interventions (2006) Archives of Disease in Childhood Vol 91 Pg 740-743

Charles $\mathrm{M}$ and Horwath J. Investing in Interagency Training to Safeguard Children: An Act of Faith or an Act of Reason? (2009) Children and Society Vol 23 Issue 5 Pg 364-376

Department for Education. Working together to safeguard children: Statuary guidance on inter0agency working to safeguard and promote the welfare of children (2018). Available at: https://www.gov.uk/government/publications/working-together-to-safeguard-children--2 Accessed June 2019

Fish S, Munro E, Bairstow S. SCIE Report 19: Learning together to safeguard children: developing a multi-agency systems approach for case reviews (2008) Social Care Institute for Excellence. 
General Medical Council. Protecting children and young people (2012 updated 2018). Available at: https://www.gmc-uk.org/ethical-guidance/ethical-guidance-for-doctors/protecting-children-andyoung-people

Harris JC, Balmer RC, Sidebotham PD. British Society of Paediatric Dentistry: a policy document on dental neglect in children (2009) International Journal Paediatric Dentistry Vol 23 Issue 1 Pg 64-71

Harris JC, Elcock C, Sidebotham PD, Welbury RR. Safeguarding children in dentistry: 1. Child protection training, experience and practice of dental professionals with an interest in paediatric dentistry (2009) British Dental Journal Vol 206 Pg 409-414

Harris JC, Elcock C, Sidebotham PD, Welbury RR. Safeguarding children in dentistry: 2. Do paediatric dentists neglect child dental neglect? (2009) British Dental Journal Vol 260 Pg 465-470

Jackson B \& Tomson M (2017) Embedding a sustainable skills-based safeguarding children course across multiple postgraduate general practice training programmes, Education for Primary Care, 28:1, 59-62, DOI: 10.1080/14739879.2016.1250608

Laming (2003) The Victoria Climbie Inquiry: report of an inquiry by Lord Laming. Available at: https://www.gov.uk/government/publications/the-victoria-climbie-inquiry-report-of-an-inquiry-bylord-laming. Accessed June 2019.

Laming. The protection of children in England: A progress report (2009) Available at: https://www.gov.uk/government/publications/the-protection-of-children-in-england-a-progressreport Accessed June 2019

Laud, A, Gizani S, Maragkou S, Welbury R, Papagiannoulis. Child protection training, experience, and personal views of dentists in the prefecture of Attica, Greece (2013) International Journal Paediatric Dentistry Vol 23 Issue 1 Pg 64-71

Lazenbatt A, Freeman R. Recognizing and reporting child physical abuse: a survey of primary healthcare professionals (2006) Journal Advanced Nursing Vol 56 Pg 227-236

NSPCC Child protection plan statistics (2019). Available at: https://learning.nspcc.org.uk/researchresources/child-protection-plan-register-statistics/ Accessed June 2019.

Public Health England. Safeguarding in general dental practice: A toolkit for dental teams. Available at:

https://assets.publishing.service.gov.uk/government/uploads/system/uploads/attachment data/file /791681/Guidance for Safeguarding in GDP.pdf Accessed June 2019.

Royal College of Nursing and Royal College of General Practice. Safeguarding children and young people: roles and competencies for healthcare staff (2019) Available at: www.rcn.org.uk/clinicaltopics/safeguarding Accessed June 2019

Welbury RR, MacAskill SG, Murphy JM, et al . General dental practitioners' perception of their role within child protection: a qualitative study (2003) European Journal Paediatric Dentistry Vol 4 Pg 8995 
Wyllie E \& Batley K (2019) Skills for safe practice - A qualitative study to evaluate the use of simulation in safeguarding children teaching for pre-registration children's nurses. Nurse Educ Pract Vol 34 p85-89 doi: 10.1016/j.nepr.2018.11.009 